\title{
Syncope: experience at a tertiary care hospital in Karachi, Pakistan
}

\author{
Muhammad Junaid Patel • Nadeem Ullah Khan • \\ Abdul Jawwad Samdani • Muhammad Furqan • \\ Aamir Hameed • Muhammad Shoaib Khan • \\ Syed Imran Ayaz $\cdot$ Muhammad Omer Jamil
}

Received: 17 January 2008 / Accepted: 17 February 2008/Published online: 6 May 2008

(C) Springer-Verlag London Ltd 2008

\begin{abstract}
Introduction Our aim was to determine the characteristics of patients presenting with syncope at a tertiary care hospital in Karachi, Pakistan.

Methods A review of medical records was conducted retrospectively at the Department of Medicine, Aga Khan University Hospital, Karachi. Patients aged 16 and above, admitted from January 2000 to December 2005 with the diagnosis of syncope made by the attending physician were included.

Results A total of 269 patients were included ( $75 \%$ males, mean age: 57.4 years). Neurogenic (vasovagal) syncope was the most common cause (47\%), followed by cardiogenic syncope (18\%) and orthostatic syncope (9\%). A total of $24 \%$ were discharged undiagnosed. Twenty patients (7.4\%) did not have any prodrome. Common prodromal symptoms included dizziness (61\%), sweating (25\%), palpitations (19\%), nausea/ vomiting (19\%) and visual symptoms (17\%). The distribution of symptoms according to cause of syncope revealed only breathlessness to be significantly associated with cardiogenic syncope $(p=0.002)$. Most patients with cardiogenic syncope
\end{abstract}

Disclaimer: The views expressed in this paper are those of the author(s) and not those of the editors, editorial board or publisher.

M. J. Patel $\cdot$ N. U. Khan $(\varangle) \cdot$ A. J. Samdani $\cdot$ M. Furqan

A. Hameed $\cdot$ M. S. Khan - S. I. Ayaz $\cdot$ M. O. Jamil

Section of Emergency Medicine, Department of Medicine,

Aga Khan University Hospital,

Stadium Road, P.O. Box 3500, Karachi 74800, Pakistan

e-mail: nadeemullah.khan@aku.edu

M. J. Patel

e-mail: junaid.patel@aku.edu were aged above $40(98 \%, p<0.001)$, had coronary artery disease $(72 \%, p<0.001)$ and abnormal electrocardiogram at presentation $(92 \%, p<0.001)$.

Conclusion Despite differences in burden of diseases, our findings were similar to those of published syncope literature. Further studies are needed to develop a protocol to expedite the evaluation and limit the work-up and admission in low-risk patients.

Keywords Syncope $\cdot$ Vasovagal $\cdot$ Cardiogenic $\cdot$ Pakistan

\section{Introduction}

Syncope is a common problem accounting for $3 \%$ of emergency department (ED) visits [1], 1-6\% of hospital admissions [2] and affecting 6 per 1,000 people per year [3]. The aetiologies of syncope vary from benign conditions like neurogenic (vasovagal) syncope to serious lifethreatening cardiac arrhythmias. In addition, falls as a result of syncope lead to $16-35 \%$ of hip fractures in elderly patients $[4,5]$. Evaluation of syncope and its appropriate management is imperative, but the exact cause remains unclear in many cases even after administering extensive work-up [6, 7].

In Pakistan there is a paucity of data available regarding syncope. As the burden of diseases in Pakistan differs from Western countries [8], there might be a possibility of difference in major causes of syncope and factors associated with it. The objective of the study was to determine the characteristics of patients admitted to the hospital for syncope to a tertiary care hospital in Karachi, Pakistan. 


\section{Methods}

Study design

Retrospective chart review.

Study population

This single-centre study was conducted in the Department of Medicine at Aga Khan University Hospital (AKUH), Karachi. It is a private, fee for service, urban tertiary care teaching hospital with 500 inpatient beds. The ED at AKUH has an annual census of approximately 40,000 patients from both adult and paediatric populations.

Charts of patients admitted under the diagnosis of "syncope" or "collapse" (made by the attending physician) were selected by a computerized data-based system from all hospital admissions during the period starting from January 2000 to December 2005. Records of patients older than 16 were reviewed. We determined the specific causes of syncope by the final diagnosis made by the attending physician and categorized them into (1) neurogenic (vasovagal), (2) cardiogenic, (3) unknown origin, (4) orthostatic and (5) psychogenic syncope.

Anyone who presented to the ED with transient loss of consciousness (TLOC) underwent evaluation for syncope. Initial evaluation included history, physical exam, orthostatic blood pressure and heart rate measurement and a 12-lead ECG with or without echocardiogram. If the initial evaluation identified the TLOC as syncope, specific tests were carried out to identify the underlying cause in the next step. We considered patients with age $>60$ years, prior history of coronary heart disease, hypertension, diabetes mellitus and abnormal ECG at presentation to have high risk factors.

\section{Statistical analysis}

During chart review we recorded the demographic details, presenting symptoms, precipitating factors, past medical history, drug history and results of investigations. For analysis, these variables were entered into the Statistical Package for Social Sciences (SPSS) version 14. Means were calculated for continuous variables and frequencies for categorical variables. A cross-tab was applied to compare different variables and $p$ values were calculated using the chi-square test.

The Institutional Ethics Review Committee approved the protocol of this research project.

\section{Results}

There were 358 patients during the study period who were admitted with the diagnosis of "syncope" or "collapse".
Records of 317 (89\%) patients were found, of which 48 $(13 \%)$ patients were excluded as they were found to have a "non-syncopal" aetiology for their symptoms. This left 269 patients for final analysis. Among these, 75.5\% $(n=203)$ were male. As per the nature of the problem most of the patients $(93 \%)$ were admitted to the hospital through the ED. The mean age of the study population was 57.4 years. Fortysix percent $(n=124)$ of patients were admitted to the general medical ward and 145 patients were admitted to the high dependency unit (monitored/telemetry beds). The median length of hospital stay was 2.0 days [interquartile ranges: 1.25 (25), 2.0 (50), 3.0 (75)]. The mean length of stay for patients with cardiogenic syncope was double (4 days) that of patients with neurogenic (vasovagal) syncope (2 days). Eighty-three percent $(n=224)$ of patients were admitted under the care of the cardiology service, $17 \%(n=45)$ under general medicine while one patient was admitted under the neurology service. The most commonly associated comorbid condition of our study population was hypertension (58\%), followed by coronary artery disease (44\%), hyperlipidaemia (33\%) and diabetes mellitus (27\%).

The frequencies of commonly associated prodromal symptoms in our study population were: dizziness $(61 \%)$, sweating (25\%), palpitation (19\%), nausea/vomiting (19\%), visual symptoms (transient blackouts/blurred vision, haloes around light) (17\%) and breathlessness (11\%). The frequencies of these symptoms according to cause of syncope and precipitating factor are described in Table 1. Regarding the position of the patient at the time of onset of syncope, $82 \%$ developed it while standing and sitting, less than $5 \%$ developed it while lying down and in $13 \%$ of the cases, the patient's position could not be determined from the records. The most common investigation done was an electrocardiogram (96\%), which was abnormal in $13 \%$. The ECG abnormalities included heart blocks, tachyarrhythmias or bradyarrhythmias and ischaemic changes. Of 38, 15 (39\%) head-up tilt tests were positive. The diagnostic yield of different tests performed is shown in Table 2.

Neurogenic (vasovagal) syncope was the most common type $(46.8 \%)$ regardless of the age, followed by cardiogenic $(18 \%)$, orthostatic $(9 \%)$ and psychogenic syncope $(1 \%)$. Twenty-four percent were discharged with the diagnosis of "syncope of unknown origin". Table 1 depicts the cause of syncope by age group. Most of our patients had syncope for the first time in their life while one fourth $(n=67)$ of our patients presented with recurrence. Among those who had recurrence, $36 \%$ of patients were diagnosed with cardiogenic syncope, $25 \%$ with syncope of unknown origin, $22 \%$ with neurogenic syncope and $15 \%$ with orthostatic hypotension. Among 145 patients who were in monitored beds, only $19 \%$ had the diagnosis of cardiogenic syncope, and $18 \%$ of patients admitted to a general medical ward were diagnosed with cardiogenic syncope. We also noticed that 
Table 1 Distributions of symptoms, precipitating factors, age and previous history of syncope across various causes of syncope

\begin{tabular}{|c|c|c|c|c|c|}
\hline & \multicolumn{5}{|l|}{ Causes of syncope } \\
\hline & $\begin{array}{l}\text { Vasovagal }(n=126) \\
n(\%)\end{array}$ & $\begin{array}{l}\text { Unknown }(n=64) \\
n(\%)\end{array}$ & $\begin{array}{l}\text { Cardiogenic }(n=50) \\
n(\%)\end{array}$ & $\begin{array}{l}\text { Orthostatic }(n=26) \\
n(\%)\end{array}$ & $\begin{array}{l}\text { Psychogenic }(n=3) \\
n(\%)\end{array}$ \\
\hline \multicolumn{6}{|l|}{ Symptoms } \\
\hline Dizziness & 87 (69) & $40(62)$ & $23(46)$ & $12(48)$ & $3(100)$ \\
\hline Visual symptoms & $33(26)$ & 9 (14) & $4(8)$ & $10(20)$ & $1(33)$ \\
\hline Nausea & $27(21)$ & $12(18)$ & $10(20)$ & $9(33)$ & $1(33)$ \\
\hline Palpitation & $23(18)$ & $12(18)$ & $10(20)$ & $9(33)$ & $1(33)$ \\
\hline Sweating & $26(21)$ & $11(17)$ & $6(12)$ & $6(24)$ & $1(33)$ \\
\hline Chest pain & $11(9)$ & $11(09)$ & $4(08)$ & $3(12)$ & $0(0)$ \\
\hline Shortness of breath & $13(10)$ & $5(08)$ & $14(28)$ & $1(4)$ & $0(0)$ \\
\hline \multicolumn{6}{|l|}{ Precipitating factors } \\
\hline Defecation/micturition & $28(22)$ & $11(17)$ & $9(18)$ & $3(12)$ & $1(33)$ \\
\hline Exertion & $18(14)$ & $12(19)$ & $7(14)$ & $3(12)$ & $1(33)$ \\
\hline Office work & $6(5)$ & $5(8)$ & $8(16)$ & $4(15)$ & $0(0)$ \\
\hline Standing after sleep & $14(11)$ & $6(9)$ & $7(14)$ & $6(23)$ & $0(0)$ \\
\hline Eating & $9(7)$ & $10(16)$ & $5(10)$ & $1(4)$ & $0(0)$ \\
\hline Unknown & $51(40)$ & $20(31)$ & $14(28)$ & $9(35)$ & $1(33)$ \\
\hline \multicolumn{6}{|l|}{ Age groups } \\
\hline $16-40$ years $(33 / 269)$ & $23(18)$ & $5(8)$ & $1(2)$ & $3(12)$ & $1(33)$ \\
\hline $41-65$ years $(146 / 269)$ & $71(56)$ & $37(58)$ & $22(44)$ & $14(54)$ & $2(67)$ \\
\hline 66 and above $(90 / 269)$ & $32(25)$ & $22(34)$ & $27(54)$ & $9(34)$ & $0(0)$ \\
\hline \multicolumn{6}{|c|}{ Previous history of syncope } \\
\hline Yes & $28(22)$ & $16(25)$ & $18(36)$ & $4(15)$ & $1(33)$ \\
\hline No & $98(78)$ & $48(75)$ & $32(64)$ & $22(85)$ & $2(66)$ \\
\hline
\end{tabular}

$48 \%(n=60)$ of patients with neurogenic syncope were admitted to the telemetry unit and more than half $(58 \%)$ of them had no high risk factors. Sixty-nine percent had troponin I measured, $46 \%$ had echocardiography, 20\% exercise tolerance test, 11\% Holter monitoring, 9\% electroencephalogram, $6 \%$ magnetic resonance imaging of the brain, $6 \%$ computed tomography scan of the brain, $6 \%$ myocardial perfusion scan and $3 \%$ dobutamine stress

Table 2 Non-invasive tests along with their diagnostic yield in the study population

\begin{tabular}{ll}
\hline Diagnostic test & $n^{\mathrm{a} / N^{\mathrm{b}}(\%)}$ \\
\hline Electrocardiogram & $34 / 258(13)$ \\
Troponin I & $4 / 180(2)$ \\
Echocardiogram & $13 / 92(7)$ \\
Holter monitoring & $6 / 46(13)$ \\
Head-up tilt test & $15 / 38(39)$ \\
Stress test & $4 / 28(14)$ \\
EEG & $0 / 22(0)$ \\
Magnetic resonance imaging & $0 / 18(0)$ \\
Myocardial perfusion scan & $3 / 16(19)$ \\
Dobutamine echo & $1 / 11(9)$ \\
Head computed tomography scan & $0 / 10(0)$ \\
Carotid Doppler & $0 / 7(0)$
\end{tabular}

${ }^{a}$ Number of patients with positive diagnostic test

${ }^{\mathrm{b}}$ Number of patients who underwent the test echocardiography. Similarly $64 \%$ of patients with "syncope of unknown origin" were admitted to monitored beds and $50 \%$ of them had no significant risk factors; and they had multiple investigations that were negative. The average cost of evaluation and stay was estimated to be Pakistani rupees 20,000 (US \$350) per patient including emergency department charges, hospitalization charges, subspecialty consultations, baseline investigations and specific tests.

We also found that patients with cardiogenic syncope had a significantly higher frequency of coronary artery disease (72\%, $p<0.001)$, abnormal ECG at presentation $(92 \%$,

Table 3 Association of risk factors with different causes of syncope

\begin{tabular}{ll}
\hline Risk factors & $n^{\mathrm{a}} / N^{\mathrm{b}}(\% \& p$ values $)$ \\
\hline History of coronary artery disease (CAD) & \\
Cardiogenic syncope & $36 / 50(72 \&<0.001)$ \\
Vasovagal syncope & $40 / 126(32 \&=0.707)$ \\
Syncope of unknown origin & $32 / 64(50 \&=0.247)$ \\
Orthostatic syncope & $11 / 26(42 \&=0.500)$ \\
Abnormal ECG at time of presentation & \\
Cardiogenic syncope & $46 / 50(92 \&<0.001)$ \\
Vasovagal syncope & $57 / 126(45 \&=0.186)$ \\
Syncope of unknown origin & $32 / 64(50 \&=0.632)$ \\
Orthostatic syncope & $18 / 26(69 \&=0.245)$
\end{tabular}

${ }^{\mathrm{a}}$ Number of patients found in each category

${ }^{\mathrm{b}}$ Total number of patients with the same type of syncope 
$p<0.001)$ and age above $40(98 \%, p<0.001)$. The risk factor profile of different types of syncope is shown in Table 3.

\section{Discussion}

Most of the published reports of syncope evaluation have been from developed countries. In this regard the current manuscript provides important information that adds to knowledge about patients presenting with syncope in a developing country in South Asia, with limited health care resources.

Our study highlights the features of syncope and their frequencies in hospitalized patients in Pakistan. We had a higher proportion of males presenting with syncope; the possible cause of this observation is either a cultural norm of our society that prefers to bring sick men to the hospital earlier than sick women. Other studies have not demonstrated such a marked gender difference [9-11].

According to the literature, prodromal symptoms of nausea/vomiting are more common with neurogenic syncope $[10,12]$; however, we did not observe such a difference in our patients. Similarly chest pain, sweating and palpitation, which point towards a cardiac aetiology, were found to be equally distributed in both neurogenic and cardiac aetiologies. Calkins et al. [13] also observed that symptoms such as palpitation, nausea and diaphoresis are not significantly helpful in ascertaining the diagnosis of syncope. Similarly De Graf et al. [10] concluded that specific symptoms cannot predict the cause of neurogenic syncope, rather it is the number of prodromal symptoms. Dizziness was prevalent in our patients, and more common in patients with neurogenic and syncope of unknown aetiology. Because of its vague nature, dizziness was reported differently in previous studies. Sloane et al. [14] found that $70 \%$ of their subjects had dizziness, lightheadedness or a sensation of impending syncope, but dizziness alone was observed in $66 \%$ of their patients. Graham et al. [15] said dizziness is relatively less common and better defined than "light-headedness". In our study, shortness of breath was the only symptom that was significantly $(p<0.05)$ associated with cardiogenic syncope. Of note, shortness of breath is also one of the prognostic factors in the San Francisco Syncope Study [16]. We conclude that presenting symptoms per se do not help in ascertaining the cause of syncope except shortness of breath.

Neurogenic syncope was the most common cause of syncope in our patients regardless of their age, but its incidence decreased with age. In contrast, cardiogenic, orthostatic and syncope of unknown aetiology follow the opposite trend. Peak frequencies of these occur after the age of 50 years. Suzuki et al. [9] and Alboni et al. [17] reported similarly and Graf et al. [10] stated that age could be one of the predictors of the cause of syncope. Increasing incidence of cardiogenic syncope with age may be because of the higher incidence of ischaemic and structural heart diseases in the elderly. Likewise increasing frequency of orthostatic syncope with advancing age is possibly explained by increased intake of multiple drugs that either affect the autonomic nervous system or deplete intravascular volume.

An important problem that emergency physicians face while dealing with patients with syncope is the decision regarding their disposition. Soteriades et al. [11] and Kapoor [18] concluded that patients with cardiac syncope are at higher risk of death from any cause while vasovagal syncope pursues a benign course. This emphasized that patients with cardiogenic syncope need hospitalization, but ascertaining the cause of syncope in most of the cases at the time of presentation is very difficult. This led investigators to find out the prognostic risk factors that can help in selection of those patients who require hospital admission. Getchell et al. [19] showed that mortality in patients with syncope increases with increase in number of comorbid conditions and age. Colivicchi et al. [20] and Martin et al. [7] also demonstrated that older age, lack of prodromal symptoms, history of cardiovascular disease (especially heart failure and ventricular arrhythmias) and abnormal ECG at presentation in patients with syncope are associated with an increase in 1-year mortality. Patients with cardiogenic syncope in our study possessed many of these prognostic risk factors in a significantly higher proportion than patients with other types of syncope. Therefore, if the diagnosis about specific type of syncope is not clear in the ED, these risk factors will help to decide who needs hospital admission.

We found that $64 \%(n=41)$ of patients with "syncope of unknown origin" had been admitted to beds with cardiac monitoring and $50 \%$ of them had no risk factors, i.e. age below 60 , no history of coronary artery disease and normal ECG at presentation. These patients had multiple investigations done, which were negative. Similarly half of the patients with neurogenic (vasovagal) syncope were admitted to specialized units and $75 \%$ of these had no history of coronary artery disease or abnormal ECG at presentation and many were aged below 60 but still underwent extensive evaluation. In addition Table 2 also shows that many patients had low-yield neurological tests.

\section{Limitations}

Several important limitations must be acknowledged in our retrospective study. First, this study only includes patients who were admitted to the hospital for syncope, which is why there were only 269 in the final cohort. No data on those who were discharged from the emergency department or left against medical advice are presented. 
Second, our hospital is a private, fee for service, urban, tertiary care centre, leading to selection bias towards urban and middle and upper income groups.

Finally, some individuals were admitted for evaluation of sudden and transient loss of consciousness, under the diagnosis of some established cause rather than syncope and thus escaped our inclusion criteria and never showed up in analysis.

\section{Conclusion}

Syncope is a relatively common medical emergency. It poses risk to the patients, hassle to the attendants, stress to the medical practitioner and burden to the health care system in general. It is essential to diagnose it right and to initiate the treatment as soon as possible. By the same token, the diagnostic approach should be cost-effective especially in a third-world country like ours. Our study results are similar to those of the existing literature. Prodromal symptoms were not found to contribute in establishing the cause of syncope. Patients with age above 40 , prior history of coronary artery disease, associated shortness of breath and abnormal ECG at presentation were more prone to have the cardiogenic type. Further studies are needed to develop a protocol to expedite the evaluation and limit the work-up and admission in low-risk patients.

\section{References}

1. Schaal SF, Nelson SD, Boudoulas H, Lewis RP (1992) Syncope. Curr Probl Cardiol 17(5):205-264

2. Maisel WH, Stevenson WG (2002) Syncope: getting to the heart of the matter. N Engl J Med 347:931-933

3. Calkins H, Byrne M, el-Atassi R, Kalbfleisch S, Langberg JJ, Morady F (1993) The economic burden of unrecognized vasodepressor syncope. Am J Med 95(5):473-479
4. Chen LY, Shen WK, Mahoney DW, Jacobson SJ, Rodeheffer RJ (2006) Prevalence of syncope in a population aged more than 45 years. Am J Med 119(12):1088.e1-1088.e7

5. Brady PA, Shen WK (1999) Syncope evaluation in the elderly. Am J Geriatr Cardiol 8:115-124

6. Ammirati F, Colivicchi F, Santini M (2000) Diagnosing syncope in clinical practice. Implementation of a simplified diagnostic algorithm in a multicentre prospective trial - the OESIL 2 study (Osservatorio Epidemiologico della Sincope nel Lazio). Eur Heart J 21(11):935-940

7. Martin TP, Hanusa BH, Kapoor WN (1997) Risk stratification of patients with syncope. Ann Emerg Med 29(4):459-466

8. Nishtar S (2004) The National Action Plan for the Prevention and Control of Non-communicable Diseases and Health Promotion in Pakistan-prelude and finale. J Pak Med Assoc 54(12 Suppl 3):S1-S8

9. Suzuki T, Matsunaga N, Kohsaka S (2006) Diagnostic patterns in the evaluation of patients hospitalized with syncope. Pacing Clin Electrophysiol 29:1240-1244

10. Graf D, Schlaepfer J, Gollut E et al (2008) Predictive models of syncope causes in an outpatient clinic. Int J Cardiol 123:249-256

11. Soteriades ES, Evans JC, Larson MG et al (2002) Incidence and prognosis of syncope. N Engl J Med 347:878-885

12. Brignole M (2007) Diagnosis and treatment of syncope. Heart 93:130-136

13. Calkins H, Shyr Y, Frumin H, Schork A, Morady F (1995) The value of the clinical history in differentiation of syncope due to ventricular tachycardia, atrioventricular block and neurocardiogenic syncope. Am J Med 98(4):365-373

14. Sloane PD, Linzer M, Pontnen M, Devine GW (1991) Clinical significance of a dizziness history in medical patients with syncope. Arch Intern Med 151(8):1625-1628

15. Graham LA, Kenny RA (2001) Clinical characteristics of patients with vasovagal reaction presenting as unexplained syncope. Europace 3:141-146

16. Quinn JV, Stiell IG, McDermott DA, Selleres KL, Kohn MA, Wells GA (2004) Derivation of the San Francisco Syncope Rule to predict patients with short-term serious outcomes. Ann Emerg Med 43(2):224-232

17. Alboni P, Brignole M, Menozzi C et al (2001) Diagnostic value of history in patients with syncope with or without heart disease. J Am Coll Cardiol 37(7):1921-1928

18. Kapoor WN (1990) Evaluation and outcome of patients with syncope. Medicine (Baltimore) 69:160-175

19. Getchell WS, Larsen GC, Morris CD, McAnulty JH (1999) Epidemiology of syncope in hospitalized patients. J Gen Intern Med 14(11):677-687

20. Colivicchi F, Ammirati F, Melina D et al (2003) Development and prospective validation of a risk stratification system for patients with syncope in the emergency department: the OESIL risk score. Eur Heart J 24:811-819 